December 13, 2018

DAMTP-2010-13

\title{
The Force Between Giant Magnons
}

\author{
Nick Dorey and Rui F. Lima Matos \\ DAMTP, Centre for Mathematical Sciences \\ University of Cambridge, Wilberforce Road \\ Cambridge CB3 0WA, UK
}

\begin{abstract}
We compute the force and torque between well-separated, slowly-moving Giant Magnons with arbitrary orientations on $S^{5}$. We propose an effective Hamiltonian for Giant Magnons in this regime.
\end{abstract}




\section{Introduction}

An interesting feature of field theory is that classical soliton solutions give rise to particle-like states after quantisation $[1,2,3]$. The resulting particles can often be usefully described by an effective Lagrangian or Hamiltonian. Famous examples of this phenomenon occur in the context of sineGordon Thirring duality [4], Olive-Montonen duality of $\mathcal{N}=4$ SUSY Yang-Mills theory [5] and the role of Skyrmions as baryons in large- $N_{c}$ QCD [6]. An interesting recent example has emerged in the context of the AdS/CFT correspondence where certain solitons on the worldsheet theory of the string on $A d S_{5} \times S^{5}$, known as Giant Magnons [7], are dual to the elementary excitations of the gauge theory spin chain. Finding an effective Hamiltonian description of these solitons is an interesting problem which should be important in the context of string quantisation. In the case of uncharged Giant Magnons moving on the same $S^{2}$ submanifold of $S^{5}$, the dynamics is closely related to that of sine-Gordon solitons and an effective Hamiltonian was proposed in [8]. Here we will address this problem in the more general case of charged Giant Magnons with arbitrary orientation on $S^{5}$. However we will solve the problem only in the case where the Giant Magnons are well-separated and slowly moving.

Here we will employ a simple approach to the problem due to Manton [9] which is available only for integrable field equations. We illustrate the method using the sine-Gordon equation,

$$
\frac{\partial^{2} \phi}{\partial t^{2}}-\frac{\partial^{2} \phi}{\partial x^{2}}=-\frac{m}{\beta} \sin \beta \phi
$$

as an example. This has a soliton solution of the form,

$$
\phi_{K}(x, t)=4 \tan ^{-1}[\exp (\gamma(v) m(x-v t))]
$$

where $\gamma(v)=1 / \sqrt{1-v^{2}}$ is the Lorentz factor. The soliton or kink interpolates between the vacuum values $\phi=0$ and $\phi=2 \pi / \beta$ and has a well defined position $X(t)=v t$ where the field takes the midpoint value $\phi=\pi / \beta$. The kink moves at constant velocity $v$ and behaves like a relativistic particle of mass $M=8 \mathrm{~m} / \beta$. A corresponding anti-kink solution is obtained by $\phi \rightarrow-\phi$ with an appropriate choice for the branch of the inverse tangent.

We now consider a configuration which contains both a kink and an anti-kink. A static configuration of this sort only solves the equation of motion in the limit of infinite separation. However, the sine-Gordon equation, being integrable, has an exact solution $\phi_{K \bar{K}}$ describing the scattering of a kink with an anti-kink. In the center of mass frame this is given as;

$$
\tan \left(\frac{\beta \phi_{K \bar{K}}}{4}\right)=\frac{1}{v} \frac{\sinh (\gamma(v) v m t)}{\cosh (\gamma(v) v x)}
$$

This solution asymptotes to a linear superposition of a kink and anti-kink, with velocities $\pm v$ respectively, at very early and late times $t \rightarrow \pm \infty$. As before we define the worldline of the kink 
as a solution $x=X(t)$ of $\phi(x, t)=\pi / \beta$ or,

$$
v \cosh (\gamma(v) v X)=\sinh (\gamma(v) v m t)
$$

For non-relativistic velocities $v<<1,(1)$ has an approximate solution at late times of the form,

$$
X(t)=-\frac{1}{m} \log v+v t-\frac{1}{m} \exp (-2 v m t)
$$

The first two terms correspond to the asymptotic free motion of the kink. The final term gives rise to an exponentially small acceleration,

$$
\ddot{X}=-4 m \exp (-2 m X)
$$

which can be interpreted in terms of an attractive potential between the kink and anti-kink, separated by a distance $s=2 X$,

$$
\mathcal{V}(s)=-\frac{32 m}{\beta^{2}} \exp (-m s)
$$

or force given by $F=-\partial \mathcal{V} / \partial s$. Finally the effective Hamiltonian describing the slow motion of both the kink and an anti-kink with positions $X_{K}$ and $X_{\bar{K}}$ respectively and conjugate momenta $P_{i}=M \dot{X}_{i}$ for $i=K, \bar{K}$ takes the form,

$$
\mathcal{H}_{K \bar{K}}=\frac{P_{K}^{2}}{2 M}+\frac{P_{\bar{K}}^{2}}{2 M}+\mathcal{V}\left(\left|X_{K}-X_{\bar{K}}\right|\right)
$$

with potential $\mathcal{V}$ given in (3). This result agrees with other approaches to determining the effective Hamiltonian at large separation. The method of $[10,11]$ involves studying the motion of poles of the analytically continued energy density ${ }^{1}$. One may also proceed by constructing static multisoliton solutions by allowing discontinuities on the spatial derivatives of the fields across the particle positions $[12,13]$. While the first of these requires the knowledge of explicit mulit-soliton solutions, the second method does not, thus being also applicable to non-integrable theories. Finally, for this system there is an exact Hamiltonian description given by the two-body Ruijsenaars-Schneider model [14], with a Hamiltonian which reduces to (3) for low velocity and large separation ${ }^{2} \mid X_{K}-$ $X_{\bar{K}} \mid>>m^{-1}$.

In this paper we will perform an analogous calculation to derive an effective Hamiltonian for two well-separated, slowly moving Giant Magnons. In addition to linear momenta $P^{(1)}$ and $P^{(2)}$, canonically conjugate to the soliton positions $X^{(1)}$ and $X^{(2)}$, these solitons also carry conserved

\footnotetext{
${ }^{1}$ This approach does not yield a Hamiltonian description of the motion for generic separations [14].

${ }^{2}$ Note however that the naive definition (1) of the soliton worldline used above will not reproduce the correct Hamiltonian description except in this regime.
} 
$U(1)$ charges $Q^{(1)}$ and $Q^{(2)}$ corresponding to internal motion with respect to angular coordinates $\Theta^{(1)}$ and $\Theta^{(2)}$. Each soliton also has additional coordinates describing its orientation on $S^{5}$. Starting from an appropriate two-soliton solution of the string $\sigma$-model, we compute the force and torque for dyonic giant magnons, each with arbitrary polarisations in $S^{5}$, in the large-separation regime where $|\Delta X| \equiv\left|X^{(2)}-X^{(1)}\right|>>1$, and where both linear and internal soliton motion is slow. These latter conditions correspond to momenta $P^{(1)}, P^{(2)}$ and charges $Q^{(1)}, Q^{(2)}$ of order one in the regime of large 't Hooft coupling $\lambda=g_{Y M}^{2} N>>1$. The resulting effective Hamiltonian takes the form:

$$
\mathcal{H}=2 M-\frac{\left[P^{(1)}\right]^{2}}{2 M}-\frac{\left[P^{(2)}\right]^{2}}{2 M}+\frac{\left[Q^{(1)}\right]^{2}}{2 M}+\frac{\left[Q^{(2)}\right]^{2}}{2 M}+\mathcal{V}\left(|\Delta X|, \Theta^{(1)}, \Theta^{(2)}\right),
$$

where $M=\sqrt{\lambda} / \pi$ is the mass of each soliton and the two-body potential is shown to have the form,

$$
\begin{gathered}
\mathcal{V}\left(|\Delta X|, \Theta^{(1)}, \Theta^{(2)}\right)=\frac{\sqrt{\lambda}}{\pi} f\left(\Theta^{(1)}, \Theta^{(2)}\right) e^{-|\Delta X|}, \\
f\left(\Theta^{(1)}, \Theta^{(2)}\right)=K^{+} \cos \left(\Theta_{1}+\Theta_{2}\right)+K^{-} \cos \left(\Theta_{1}-\Theta_{2}\right), \\
K^{ \pm}=K^{ \pm}\left(\left\|\overrightarrow{J_{1}}\right\|,\left\|\overrightarrow{J_{2}}\right\|, \vec{J}_{1} \cdot \overrightarrow{J_{2}}\right) .
\end{gathered}
$$

The coefficients $K^{ \pm}$are given explicitly in (51) as functions of the $S O(4)$ invariants formed from the $S^{5}$ angular momenta $\overrightarrow{J_{1}}$ and $\overrightarrow{J_{2}}$ of the two Giant Magnons.

For solitons of charge $Q^{(1)}, Q^{(2)}=0$ the two-body potential (5) reduces to the sine-Gordon result (3). This reflects the correspondence between uncharged Giant Magnons and sine-Gordon kinks induced by Pohlmeyer reduction [17] and our results are consistent with those of [8] in this case. The effective Hamiltonian for charged Giant Magnons with parallel angular momenta on $S^{5}$ was also considered in [16]. In particular, general constraints on the Hamiltonian were considered and a toy model which correctly reproduces the known analytic structure of the magnon S-matrix was proposed in this reference. Restricting our result to the case of parallel $S^{5}$ angular momenta we find that the Hamiltonian (4) has the same asymptotic form as the toy model considered in [16]. We believe our result should provide a useful starting point for the search for an exact Hamiltonian description of Giant Magnons.

\section{Giant Magnons on $\mathbb{R} \times S^{5}$}

The bosonic sector of type IIB superstring theory in the background $A d S_{5} \times S^{5}$ can be described by the following $\sigma$-model action

$$
S=\frac{\sqrt{\lambda}}{2 \pi} \int d x d t\{\overbrace{\eta^{a b} \partial_{a} Y^{\mu} \partial_{b} Y_{\mu}+\Lambda_{1}\left(Y_{\mu} Y^{\mu}+1\right)}^{A d S_{5}}+\underbrace{\eta^{a b} \partial_{a} X^{j} \partial_{b} X_{j}+\Lambda_{2}\left(X_{j} X^{j}-1\right)}_{S^{5}}\},
$$


where $(x, t)$ are the string worldsheet coordinates, $\vec{Y}, \vec{X} \in \mathbb{R}^{6}$ parametrise the string embedding,

$$
\begin{aligned}
& Y_{\mu} Y^{\mu}=-Y_{-1}^{2}-Y_{0}^{2}+Y_{1}^{2}+Y_{2}^{2}+Y_{3}^{2}+Y_{4}^{2}=1, \\
& X_{j} X^{j}=X_{1}^{2}+X_{2}^{2}+X_{3}^{2}+X_{4}^{2}+X_{5}^{2}+X_{6}^{2}=1,
\end{aligned}
$$

$\eta^{a b}$ is the (Minkowskian) string worldsheet metric in conformal gauge and $\Lambda_{1,2}$ are Lagrange multipliers that enforce (9) and (10). In what follows it will be convenient to introduce the following parametrisation of $S^{5}$,

$$
Z_{1}=X_{1}+i X_{2}, \quad Z_{2}=X_{3}+i X_{4}, \quad Z_{3}=X_{5}+i X_{6}, \quad \text { with } \quad \sum_{j=1}^{3}\left|Z_{j}\right|^{2}=1 .
$$

The action (8) has the global symmetry $S O(4,2) \times S O(6)$. Through this paper we will be interested in a subset of the solutions of the Euler-Lagrange equations for the above action, namely those restricted to the submanifold $\mathbb{R} \times S^{5} \subset A d S_{5} \times S^{5}$. We will choose the gauge $Y^{0}=t$. The generator of time translations will then be given by

$$
\Delta=i \frac{\sqrt{\lambda}}{2 \pi} \int d x \dot{Y}_{0}=i \frac{\sqrt{\lambda}}{2 \pi} \int d x
$$

and the generator of rotations in $S^{5}$ by

$$
J_{i j}=i \frac{\sqrt{\lambda}}{2 \pi} \int d x X_{[i} \dot{X}_{j]}, \quad i, j=1, \ldots, 6 .
$$

Following [7], we will consider the limit when the angular momentum $J \equiv J_{12}$ is very large and look for solutions for which the difference $E \equiv \Delta-J$ remains finite [7]. In this limit the string becomes infinitely long and we can relax the closed string boundary condition, allowing different values for the fields $Z_{j}$ at left and right infinity $x \rightarrow \pm \infty$. The simplest solution one can construct is the BMN groundstate solution [18]

$$
Z_{1}^{(B M N)}=e^{i t}, \quad Z_{2}^{(B M N)}=Z_{3}^{(B M N)}=0 .
$$

which describes the motion of a point-like string travelling along the equator $S^{1}$ of $S^{5}$ with very large angular momentum $J$. It saturates a BPS bound obeying,

$$
\Delta-J=0 .
$$

The Giant Magnon is a solitonic excitation of the BMN vacuum (14). The simplest case is the original uncharged Giant Magnon of [7] which moves on and $S^{2} \in S^{5}$. The solution takes the form,

$$
\left\{\begin{array}{l}
\left.Z_{1}=e^{i t}\left\{\gamma^{-1} \tanh \left(\gamma\left[\left(x-x_{0}\right)-V t\right]\right)\right]-i V\right\} \\
Z_{2}=\gamma^{-1} \operatorname{sech}\left(\gamma\left[\left(x-x_{0}\right)-V t\right]\right) \\
Z_{3}=0
\end{array}\right.
$$


with $V=\cos (p / 2)$ and $\gamma=\left(1-V^{2}\right)^{-\frac{1}{2}}$. The solution carries a conserved momentum $p$ which is related to the large- $x$ asymptotics of the fields according to,

$$
\lim _{x \rightarrow \pm \infty} Z_{j}\left[x, t ; p, x_{0}\right]=-i Z_{j}^{(B M N)}[t] e^{ \pm i \frac{p}{2}}, \quad j=1,2,3 .
$$

This excitation contributes a finite amount to the anomalous dimension $\Delta-J$ and has dispersion, relation,

$$
\Delta-J=\frac{\sqrt{\lambda}}{\pi} \sin \left(\frac{p}{2}\right)
$$

The solution (16) has a well-defined center,

$$
X(t)=x_{0}+V t .
$$

which moves with constant velocity $V$. The center $X(t)$ is the point where $\left|Z_{2}\right|$ is maximal which therefore solves,

$$
\partial_{x}\left|Z_{2}\left[X(t), t ; p, x_{0}\right]\right|=0 .
$$

We will adopt this definition for the position coordinate of the soliton in a more general context below. The conserved momentum $p$ introduced above is canonically conjugate to $X[7]$ and the pair $\{p, X\}$ can be regarded as the collective coordinates of this neutral Giant Magnon solution.

The above solution admits a generalisation which, in addition to linear momentum, carries a conserved $U(1)$ charge. This solution is the dyonic giant magnon of [19] (see also [20])) which corresponds to a string moving on an $S^{3}$ submanifold of $S^{5}$,

$$
\left\{\begin{array}{l}
Z_{1}=e^{i t}\left\{\sin \left(\frac{p}{2}\right) \tanh u\left(x-x_{0}, t ; r, p\right)-i \cos \left(\frac{p}{2}\right)\right\} \\
Z_{2}=\sin \left(\frac{p}{2}\right) \operatorname{sech} u\left(x-x_{0}, t ; r, p\right) e^{i\left[v\left(x-x_{0} ; r, p\right)+\theta_{0}\right]} \\
Z_{3}=0
\end{array}\right.
$$

Here we have introduced the functions

$$
\begin{aligned}
u(x, t ; r, p) & =\frac{2 r\left(1+r^{2}\right) \sin \left(\frac{p}{2}\right)}{1-2 r^{2} \cos p+r^{4}}[x-V t], \\
v(x, t ; r, p) & =\frac{2 r\left(r^{2}-1\right) \cos \left(\frac{p}{2}\right)}{1-2 r^{2} \cos p+r^{4}}\left[x-V^{-1} t\right], \\
V & =\frac{2 r}{r^{2}+1} \cos \left(\frac{p}{2}\right) .
\end{aligned}
$$

The conserved $U(1)$ charge is, $Q=\frac{\sqrt{\lambda}}{2 \pi}\left(r-r^{-1}\right),(r \in \mathbb{R})$, Setting $Q=0$ we return to the neutral Giant Magnon described in the preceding paragraph. The dispersion relation of the new solution is given by

$$
\Delta-J=\sqrt{Q^{2}+\frac{\lambda}{\pi^{2}} \sin \left(\frac{p}{2}\right)} .
$$


Its asymptotics are identical to (17),

$$
\lim _{x \rightarrow \pm \infty} Z_{j}\left[x, t ; p, x_{0}, Q, \theta_{0}\right]=-i Z_{j}^{(B M N)}[t] e^{ \pm i \frac{p}{2}}, \quad j=1,2,3 .
$$

In addition to a position $X(t)$ defined as above, the dyonic Giant Magnon has an additional degree of freedom $\Theta(t)$ corresponding to $U(1)$ rotations acting on the target space coordinate $Z_{2}$. Here $\Theta(t)$ is an angular coordinate which evolves linearly on the one-soliton solution,

$$
\Theta(t)=\theta_{0}+\omega t
$$

with angular velocity,

$$
\omega=\frac{1-r^{2}}{1+r^{2}}
$$

and is canonically conjugate to the $U(1)$ charge $Q$ The initial value $\theta_{0}$ corresponds to the argument of $Z_{2}$ at time $t=0$ evaluated at the point $x=x_{0}$. More generally we can define that phase at any other time $t$ by

$$
\Theta(t)=\arg \left\{Z_{2}\left[X(t), t ; p, x_{0}, Q, \theta_{0}\right]\right\} .
$$

The set of collective coordinates for a dyonic Giant magnon are therefore $\{p, X ; Q, \Theta\}$.

The dyonic Giant Magnon solution involves the choice of an $S^{3} \subset S^{5}$. For the solution described above this corresponds to the choice $Z_{3}=0$. We obtain more general solutions which asymptote to the same vacuum at spatial infinity by performing an $S O(4)$ rotation on the embedding coordinates $\left(X_{3}, X_{4}, X_{5}, X_{6}\right)$. Of the possible $S O(4)$ rotations, a rotation acting only on $\left(X_{5}, X_{6}\right)$ - i.e. a phase shift in $Z_{3}$ - will not change our initial solution. Similarly a rotation in the plane $\left(X_{3}, X_{4}\right)$ will simply induce a phase shift in $Z_{2}$, that can be absorbed by shifting the constant $\theta_{0}$. Thus the stabilizer in $S O(4)$ of the solution $(21)$ is $S O(2) \times S O(2)$. Inequivalent embeddings of the dyonic Giant Magnon on $S^{5}$ are therefore parametrised by the coset [20, 21],

$$
G r_{2}\left(\mathbb{R}^{4}\right)=\frac{S O(4)}{S O(2) \times S O(2)} \approx \frac{S O(3) \times S O(3)}{S O(2) \times S O(2)} \approx S_{+}^{2} \times S_{-}^{2} .
$$

We introduce polar coordinates on each of the spheres $S_{ \pm}^{2}$, by choosing a unit vectors,

$$
\vec{n}_{ \pm}=\left(\cos \left[\psi_{+} \mp \psi_{-}\right] \sin \alpha_{ \pm}, \sin \left[\psi_{+} \mp \psi_{-}\right] \sin \alpha_{ \pm}, \cos \alpha_{ \pm}\right) .
$$

Finally, performing an $S O(4)$ rotation on (21), the most general Giant Magnon solution solution in $\mathbb{R} \times S^{5}$ has then the form [21],

$$
Z_{j}=Z_{j}\left[x, t ; p, x_{0}, Q, \theta_{0}, \vec{n}_{ \pm}\right], \quad j=1,2,3
$$


with,

$$
\left\{\begin{array}{r}
Z_{1}=e^{i t}\left\{\sin \left(\frac{p}{2}\right) \tanh u\left(x-x_{0}, t ; r, p\right)-i \cos \left(\frac{p}{2}\right)\right\} \\
Z_{2}=e^{i \psi^{+}} \sin \left(\frac{p}{2}\right) \operatorname{sech} u\left(x-x_{0}, t ; r, p\right)\left[\cos \left\{v\left(x-x_{0} ; r, p\right)+\theta_{0}\right\} \cos \Delta \alpha\right. \\
\left.+i \sin \left\{v\left(x-x_{0} ; r, p\right)+\theta_{0}\right\} \cos \bar{\alpha}\right], \\
Z_{3}=e^{i \psi^{-}} \sin \left(\frac{p}{2}\right) \operatorname{sech} u\left(x-x_{0}, t ; r, p\right)\left[\cos \left\{v\left(x-x_{0} ; r, p\right)+\theta_{0}\right\} \sin \Delta \alpha\right. \\
\left.+i \sin \left\{v\left(x-x_{0} ; r, p\right)+\theta_{0}\right\} \sin \bar{\alpha}\right],
\end{array}\right.
$$

where $\bar{\alpha}=\alpha_{+}-\alpha_{-}, \Delta \alpha=\alpha_{+}+\alpha_{-}$. This solution has the asymptotics (26) and it obeys the dispersion relation (25), with $Q=\mp \operatorname{tr}\left[\left(\vec{J}^{ \pm}\right)^{2}\right]$. The angular momentum of the solution can be written in the form

$$
\begin{aligned}
\vec{J} & \equiv\left(J_{i j}\right)=\sum_{\substack{a \in\{1,2,3\} \\
k= \pm}} J_{a}^{k} \mathbf{t}_{a}^{k}, \quad i, j=3, \ldots, 6 \\
\vec{J}^{ \pm} & \equiv\left(J_{a}^{ \pm}\right)= \pm Q\left(\cos \left(2 \alpha_{ \pm}\right),-\cos \left[\psi_{+} \mp \psi_{-}\right] \sin \left(2 \alpha_{ \pm}\right), \sin \left[\psi_{+} \mp \psi_{-}\right] \sin \left(2 \alpha_{ \pm}\right)\right),
\end{aligned}
$$

with $\left\{\mathbf{t}_{a}^{ \pm}\right\}$a basis for the Lie algebra $\mathfrak{s o}(4)=\mathfrak{s u}(2) \oplus \mathfrak{s u}(2)$ which is given in the Appendix. As expected, $\vec{J}^{+}$and $\vec{J}^{-}$parametrize two copies of $S^{2}$ with the same fixed radius equal to the $U(1)$ charge $Q$. The complete set of collective coordinates of the most general Giant Magnon solution is therefore $\left\{p, X, Q, \Theta, \vec{n}_{ \pm}\right\}$. As above, $X(t)$ and $\Theta(t)$ evolve linearly according to,

$$
\begin{gathered}
X(t)=x_{0}+V[p] t, \quad \Theta(t)=\theta_{0}+\omega[Q] t \\
V[p]=\frac{\sqrt{\lambda}}{\pi} M(r)^{-1} \cos \left(\frac{p}{2}\right), \quad \omega[Q]=\frac{Q}{M(r)}, \quad M(r) \equiv E[p=\pi]=\frac{\sqrt{\lambda}}{2 \pi} \frac{1+r^{2}}{r},
\end{gathered}
$$

The remaining collective coordinates are constants of motion. The quantity $M(r)$ will play the role of the soliton mass in the non-relativistic limit.

\section{The Force and Torque between Giant Magnons}

Let us now consider a configuration containing two Giant Magnons of the form (32),

$$
\begin{aligned}
& Z_{j}^{(1)} \equiv Z_{j}\left[x, t ; p^{(1)}, x_{0}^{(1)}, Q^{(1)}, \theta_{0}^{(1)}, \vec{n}_{ \pm}^{(1)}\right] \\
& Z_{j}^{(2)} \equiv Z_{j}\left[x, t ; p^{(2)}, x_{0}^{(2)}, Q^{(2)}, \theta_{0}^{(2)}, \vec{n}_{ \pm}^{(2)}\right],
\end{aligned}
$$

separated by a distance $d=\left|x_{0}^{(1)}-x_{0}^{(2)}\right|$, at time $t=0$, and with momenta $p^{(1)}=\pi-\delta$ and $p^{(2)}=\pi+\delta$ in the centre of momentum frame and charges $Q^{(1)}=\sqrt{\lambda}\left(r_{1}-r_{1}^{-1}\right) / 2 \pi$ and $Q^{(2)}=$

$\sqrt{\lambda}\left(r_{2}-r_{2}^{-1}\right) / 2 \pi$. The effective Hamiltonian will be invariant under all global symmetries left unbroken by the BMN vacuum. Thus, without loss of generality we may fix $x_{0}^{(1)}=0$ and $x_{0}^{(2)}=-d$ 
and, by using the global $S O(4)$ symmetry, fix $\vec{n}_{ \pm}^{(1)}$ of the first soliton to be $(0,0,1)$. In other words we can choose,

$$
\left\{\begin{array}{l}
\theta_{0}^{(1)}=0, \quad \alpha_{ \pm}^{(1)}=0, \quad \psi_{ \pm}^{(1)}=0 \\
\theta_{0}^{(2)}=\theta_{0}, \quad \alpha_{ \pm}^{(2)}=\alpha_{ \pm} \quad \psi_{ \pm}^{(2)}=\psi_{ \pm}
\end{array}\right.
$$

Using the stabiliser of the first soliton solution we may further rotate the second soliton in the $Z_{3}$ plane and set $\psi_{-}=0$.

As for the sine-Gordon case discussed in the introduction, a linear superposition of two solitons does not solve the equations of motion except in the limit of infinite separation. To compute the force between these solitons we need to find an exact scattering solution $\mathcal{Z}_{j}$ which asymptotes to a configuration of two well-separated solitons, with parameters chosen as in (38) at very early and late times. By shifting the time coordinate we can choose the origin $t=0$ to lie in the early asymptotic region and demand,

$$
\mathcal{Z}_{j} \stackrel{t=0, d \rightarrow \infty}{\longrightarrow} Z_{j}^{(1)}+Z_{j}^{(2)} .
$$

The equations of motion of the $A d S_{5} \times S^{5} \sigma$-model are integrable and it is possible to construct the required two soliton scattering solution explicitly using the dressing method [20, 21]. This is accomplished in the Appendix where the resulting solution is specified by equation (67). As expected, near $x=0$ and $x=-d, \mathcal{Z}_{j}$ resembles $Z_{j}^{(1)}$ and $Z_{j}^{(2)}$ respectively. Expanding the solution around this limit to first non-trivial order we obtain,

$$
\begin{cases}\mathcal{Z}_{j} \approx Z_{j}^{(1)}+\delta Z_{j}^{(1)}\left[x, t ; \delta, Q^{(1)}, Q^{(2)}, \theta_{0}, \alpha_{ \pm}, \psi_{+}\right] e^{-D_{2}}, & \text { for }\left|x-\omega^{(1)} t\right| \sim e^{-D_{2}} \\ \mathcal{Z}_{j} \approx Z_{j}^{(2)}+\delta Z_{j}^{(2)}\left[x, t ; \delta, Q^{(1)}, Q^{(2)}, \theta_{0}, \alpha_{ \pm}, \psi_{+}\right] e^{-D_{1}}, & \text { for }\left|x+d-\omega^{(2)} t\right| \sim e^{-D_{1}}\end{cases}
$$

where

$$
D_{j} \equiv u\left(d, 0 ; r_{j}, p^{(j)}\right)=\frac{2 r_{j}\left(1+r_{j}^{2}\right)}{1-2 r_{j}^{2} \cos p^{(j)}+r_{j}^{4}} d, \quad j=1,2 .
$$

The correction terms $\delta Z_{j}^{(1)}$ and $\delta Z_{j}^{(2)}$, will alter the linear evolution of the collective coordinates for each soliton. We make the ansatz,

$$
\left\{\begin{array}{l}
X^{(1)}(t)=V^{(1)} t+\delta X^{(1)}(t) e^{-D_{2}}, \quad \Theta^{(1)}(t)=\omega^{(1)} t+\delta \Theta^{(1)}(t) e^{-D_{2}} \\
X^{(2)}(t)=x_{0}+V^{(2)} t+\delta X^{(2)}(t) e^{-D_{1}}, \quad \Theta^{(2)}(t)=\theta_{0}+\omega^{(2)} t+\delta \Theta^{(2)}(t) e^{-D_{1}}
\end{array}\right.
$$

For computing the two-body force and torque, we need to compute the corrections $\delta X^{(1)}(t)$ and $\delta \Theta^{(1)}(t)$ to the world-line of the first soliton. At early times $t \simeq 0$, the position coordinate of the soliton is determined as in (20) by,

$$
0=\partial_{x}\left|\mathcal{Z}_{2}\right| \approx \partial_{x}\left\{\left|Z_{2}^{(1)}\right|\left(1+\Re\left[\frac{\delta Z_{2}^{(1)}}{Z_{2}^{(1)}}\right] e^{-D_{2}}\right)\right\}, \quad x \approx \omega^{(1)} t+\delta X^{(1)} e^{-D_{2}},
$$


As in the sine-Gordon case studied in the introduction we expect this simple-minded definition of the worldline to be adequate only in the limit of large separation: $d \rightarrow \infty$.

The correction $\delta Z_{2}^{(1)}$ can expressed in terms of the functions

$$
u_{a} \equiv u\left(x, t, r_{j}, p^{(a)}\right), \quad v_{a} \equiv v\left(x, t, r_{1}, p^{(a)}\right), \quad a=1,2,
$$

defined for each soliton as in Equation $(23,24)$ above. The lengthy explicit expression for $\delta Z_{2}^{(1)}$ is given in Equation (75) of the Appendix. Taking the non-relativistic limit of low velocity $\delta<<1$, we then find,

$$
\partial_{x}\left|\mathcal{Z}_{2}\right|=\left(u_{1}^{\prime} \partial_{u_{1}}+u_{2}^{\prime} \partial_{u_{2}}+v_{1}^{\prime} \partial_{v_{1}}+v_{2}^{\prime} \partial_{v_{2}}\right)\left|\mathcal{Z}_{2}\right| \approx\left(u_{1}^{\prime} \partial_{u_{1}}+u_{2}^{\prime} \partial_{u_{2}}\right)\left|\mathcal{Z}_{2}\right|
$$

where prime denotes partial derivative with respect to the spacelike worldsheet coordinate $x$ and we have neglected the contribution comming from $v_{j}^{\prime}$ because these are of order $\delta$. From the explicit expression (75) for $\delta Z_{2}^{(1)}$ and (76) we have that,

$$
\partial_{u_{2}} \Re\left[\frac{\delta Z_{2}^{(1)}}{Z_{2}^{(1)}}\right]_{x=\omega^{(1)} t}=-\Re\left[\frac{\delta Z_{2}^{(1)}}{Z_{2}^{(1)}}\right]_{x=\omega^{(1)} t}=0,
$$

thus deriving, in the non-relativistic limit,

$$
\delta X^{(1)} \approx \frac{1}{u_{1}^{\prime}} \Re\left[\partial_{u_{1}}\left(\frac{\delta Z_{2}^{(1)}}{Z_{2}^{(1)}}\right)\right]_{x=\omega^{(1)} t} .
$$

As in equation (29) above, we define the internal angle $\Theta^{(1)}(t)$ of the first soliton as the phase of $Z_{2}$ evaluated at $x=X^{(1)}(t)$. In a similar fashion, by expanding the argument of $\mathcal{Z}_{2}$ in the neighbourhood of $x=\omega^{(1)} t$, for small fixed $t$, we get

$$
\delta \Theta_{1}^{(1)}(t)=\Im\left\{\frac{\delta Z_{2}^{(1)}}{Z_{2}^{(1)}}\right\}_{x=\omega^{(1)} t} .
$$

We then find, using (45) and (46) with $\delta Z_{2}^{(1)}$ given by (75) and by taking the non-relativistic limit $\delta<<1$, that

$$
\begin{aligned}
& \delta X^{(1)}(t)=-\frac{r_{1}^{2}+1}{r_{1}} e^{-\left(u_{2}+D\right)}\left\{\frac{r_{1}^{2}+r_{2}^{2}}{\left(r_{1}+r_{2}\right)^{2}} \cos \alpha_{+} \cos \alpha_{-} \cos \left(v_{1}-v_{2}-\theta_{0}-\psi_{+}\right)\right. \\
&\left.+\frac{1+r_{1}^{2} r_{2}^{2}}{\left(1+r_{1} r_{2}\right)^{2}} \sin \alpha_{+} \sin \alpha_{-} \cos \left(v_{1}+v_{2}+\theta_{0}-\psi_{+}\right)\right\} \\
& \delta \Theta_{1}^{(1)}(t)=4 r_{1} r_{2} e^{-\left(u_{2}+D\right)}\left\{\frac{1}{\left(r_{1}+r_{2}\right)^{2}} \cos \alpha_{+} \cos \alpha_{-} \cos \left(v_{1}-v_{2}-\theta_{0}-\psi_{+}\right)\right. \\
&\left.+\frac{1}{\left(1+r_{1} r_{2}\right)^{2}} \sin \alpha_{+} \sin \alpha_{-} \cos \left(v_{1}+v_{2}+\theta_{0}-\psi_{+}\right)\right\}
\end{aligned}
$$


If we further impose that the two solitons have charges $Q^{(1)}, Q^{(2)}$ of order one for $\lambda>>1$, or equivalently that the angular velocities are small, $\omega^{(j)}<<1$, we find that

$$
\begin{aligned}
& m^{(1)} \ddot{X}^{(1)} \approx-\frac{\sqrt{\lambda}}{\pi}\left\{K_{+} \cos \left(\Theta^{(1)}+\Theta^{(2)}-\psi_{+}\right)+K_{-} \cos \left(\Theta^{(1)}-\Theta^{(2)}-\psi_{+}\right)\right\} e^{\left(X^{(2)}-X^{(1)}\right)}, \\
& m^{(1)} \ddot{\Theta}^{(1)} \approx \frac{\sqrt{\lambda}}{\pi}\left\{K_{+} \cos \left(\Theta^{(1)}+\Theta^{(2)}-\psi_{+}\right)+K_{-} \cos \left(\Theta^{(1)}-\Theta^{(2)}-\psi_{+}\right)\right\} e^{\left(X^{(2)}-X^{(1)}\right)},
\end{aligned}
$$

with the factors $K_{ \pm}=K_{ \pm}\left(\vec{J}_{1}^{ \pm}, \vec{J}_{2}^{ \pm}\right)$depending only on the angular momenta $\overrightarrow{J_{1}}, \overrightarrow{J_{2}}$ of the two solitons:

$$
K_{ \pm}=-\frac{2}{J_{1} J_{2}} \frac{\left(J_{1} \pm J_{2}\right)^{2}}{\left\|\vec{J}_{1}^{-}-\vec{J}_{2}^{-}\right\|\left\|\vec{J}_{1}^{+}-\vec{J}_{2}^{+}\right\|} \sqrt{\left(J_{1} J_{2} \mp \vec{J}_{1}^{-} \cdot \vec{J}_{2}^{-}\right)\left(J_{1} J_{2} \mp \vec{J}_{1}^{+} \cdot \vec{J}_{2}^{+}\right)}
$$

By defining the effective potential

$$
\mathcal{V}\left(x, \theta_{1}, \theta_{2}\right)=\frac{\sqrt{\lambda}}{\pi}\left\{K_{+} \cos \left(\theta_{1}+\theta_{2}\right)+K_{-} \cos \left(\theta_{1}-\theta_{2}\right)\right\} e^{-x},
$$

we can write for the force $\mathcal{F}^{(1)}$ and torque $\mathcal{T}^{(1)}$ exerted by the second soliton on the first,

$$
\begin{gathered}
\mathcal{F}^{(1)} \equiv m_{1} \ddot{X}^{(1)} \approx \frac{d}{d X^{(1)}} \mathcal{V}\left(|\Delta X|, \Theta_{1}, \Theta_{2}\right), \\
\mathcal{T}^{(1)} \equiv m_{1} \ddot{\Theta}_{1} \approx-\frac{d}{d \Theta_{1}} \mathcal{V}\left(|\Delta X|, \Theta_{1}, \Theta_{2}\right),
\end{gathered}
$$

with $\Delta X=X^{(2)}-X^{(1)}$ and where we have absorbed $\psi_{+}$in the initial value of $\Theta^{(1)}$. Identical results are obtained for the force and torque on the second soliton.

For neutral Giant Magnons $\left(Q^{(1)}=Q^{(2)}=0\right)$ the force reduces to

$$
\mathcal{F}^{(1)} \approx-4 \frac{\sqrt{\lambda}}{\pi} \cos (\Delta \alpha) \cos \left(\psi_{+}\right) e^{-|\Delta X|},
$$

where $\Delta \alpha=\alpha_{+}-\alpha_{-}$. Here $\psi_{+}$is the phase difference between two solitons embedded on the same $\mathbb{R} \times S^{2}$ when we make $\alpha_{ \pm}=0\left(\psi_{+}=0\right.$ for a pair of solitons and $\psi_{+}=\pi$ for a soliton-antisoliton pair). If both solitons have the same phase, the equation of motion is simply

$$
\ddot{X}^{(1)}=-4 e^{-|\Delta X|} \text {. }
$$

We can compare this result with the sine-Gordon result (2) which can be written as,

$$
\ddot{X}_{s G}^{(1)}=-4 m e^{-m|\Delta X|},
$$

As expected the two results agree when we set the sine-Gordon mass $m$ to unity.

By expanding their exact dispersion relation (25), the energy of two free Giant Magnons in the non-relativistic regime $\left(\delta,\left|Q^{(1)}\right|,\left|Q^{(2)}\right|<<1\right)$ is given as,

$$
E_{1+2}=2 M+\frac{1}{2} M\left[\omega^{(1)}\right]^{2}+\frac{1}{2} M\left[\omega^{(2)}\right]^{2}-\frac{1}{2} M\left[V^{(1)}\right]^{2}+\frac{1}{2} M\left[V^{(2)}\right]^{2},
$$


where the effective mass (and moment of inertia) is $M=M(0)=\sqrt{\lambda} / \pi$. The leading large-distance interaction between the two solitons can be incorporated by adding the two-body potential $\mathcal{V}$ introduced above. Finally, the effective Hamiltonian describing the interaction of two dyonic giant magnons, in the regime where they are well separated and slow moving can be written as,

$$
\mathcal{H}=2 M-\frac{\left[P^{(1)}\right]^{2}}{2 m}-\frac{\left[P^{(2)}\right]^{2}}{2 m}+\frac{\left[Q^{(1)}\right]^{2}}{2 m}+\frac{\left[Q^{(2)}\right]^{2}}{2 m}+\mathcal{V}\left(|\Delta X|, \Theta^{(1)}, \Theta^{(2)}\right),
$$

where $P^{(a)}$ and $Q^{(a)}$ the canonical conjugate momenta to $X^{(a)}$ and $\Theta^{(a)}$ respectively. The corresponding Poisson brackets are,

$$
\begin{array}{r}
\left\{Q^{(a)}, Q^{(b)}\right\}=\left\{P^{(a)}, P^{(b)}\right\}=\left\{Q^{(a)}, P^{(b)}\right\}=0, \\
\left\{X^{(a)}, X^{(b)}\right\}=\left\{\Theta^{(a)}, \Theta^{(b)}\right\}=\left\{X^{(a)}, \Theta^{(b)}\right\}=0, \\
\left\{P^{(a)}, X^{(b)}\right\}=\delta_{a b}, \quad\left\{Q^{(a)}, \Theta^{(b)}\right\}=\delta_{a b}, \quad a, b=1,2,
\end{array}
$$

By construction, equations of motion following from the Hamiltonian (59) coincide with (53-54), and the expression provides the expected energy (58) when $d \rightarrow \infty$.

In [16] a toy model for the dynamics of Giant Magnons was presented. An attractive feature of the model was that the resulting S-matrix for magnon scattering had a similar analytic structure to the exact S-matrix known from the asymptotic Bethe ansatz. For two magnons aligned in the same $S^{3}$ subspace of $S^{5}$, the toy model Hamiltonian can be cast in the form

$$
\mathcal{H}_{D H M}=2 M-\Re\left\{\frac{2 \pi_{1}^{2}}{m}+\frac{2 \pi_{2}^{2}}{m}+\frac{A}{\sinh ^{2}\left(z_{1}-z_{2}\right)}\right\},
$$

for complex phase space variables $\left(\pi_{a}, z_{a}\right) \in \mathbb{C}^{2}$, with $a=1,2$. By taking $z_{a}=\frac{1}{2}\left(X^{(a)}+i \Theta^{(a)}\right)$, $\pi_{a}=\frac{1}{2}\left(P^{(a)}+i Q^{(a)}\right)$ and going to the limit of large separation $d=\left|X^{(1)}-X^{(2)}\right|>>1$, the Hamiltonian reduces to

$$
\mathcal{H}_{D H M} \approx 2 M-\frac{\left[P^{(1)}\right]^{2}}{2 M}-\frac{\left[P^{(2)}\right]^{2}}{2 M}+\frac{\left[Q^{(1)}\right]^{2}}{2 M}+\frac{\left[Q^{(2)}\right]^{2}}{2 M}+4 A \cos (\Delta \Theta) e^{-\Delta X} .
$$

In the case $A=-M=-\sqrt{\lambda} / \pi$, we see that $H_{D G M}$ coincides with the effective Hamiltonian $\mathcal{H}$ as given in (59) when the solitons have parallel angular momenta, i.e., $\vec{J}_{1}^{ \pm} \cdot \vec{J}_{2}^{ \pm}=J_{1} J_{2}=Q_{1} Q_{2}$. 


\section{Appendix}

\section{A basis for $\mathfrak{s u}(2) \oplus \mathfrak{s u} 2$}

Through this paper we have used the following basis $\left\{\mathbf{t}_{a}^{ \pm}\right\}$for the Lie algebra $\mathfrak{s u}(2) \oplus \mathfrak{s u}(2)$,

$$
\begin{aligned}
& \mathbf{t}_{1}^{-}=\left(\begin{array}{cccc}
0 & 1 & 0 & 0 \\
-1 & 0 & 0 & 0 \\
0 & 0 & 0 & 1 \\
0 & 0 & -1 & 0
\end{array}\right), \mathbf{t}_{2}^{-}=\left(\begin{array}{cccc}
0 & 0 & 0 & 1 \\
0 & 0 & 1 & 0 \\
0 & -1 & 0 & 0 \\
-1 & 0 & 0 & 0
\end{array}\right), \quad \mathbf{t}_{3}^{-}=\left(\begin{array}{cccc}
0 & 0 & 1 & 0 \\
0 & 0 & 0 & -1 \\
-1 & 0 & 0 & 0 \\
0 & 1 & 0 & 0
\end{array}\right), \\
& \mathbf{t}_{1}^{+}=\left(\begin{array}{cccc}
0 & -1 & 0 & 0 \\
1 & 0 & 0 & 0 \\
0 & 0 & 0 & 1 \\
0 & 0 & -1 & 0
\end{array}\right), \mathbf{t}_{2}^{+}=\left(\begin{array}{cccc}
0 & 0 & 0 & 1 \\
0 & 0 & -1 & 0 \\
0 & 1 & 0 & 0 \\
-1 & 0 & 0 & 0
\end{array}\right), \mathbf{t}_{3}^{+}=\left(\begin{array}{cccc}
0 & 0 & -1 & 0 \\
0 & 0 & 0 & -1 \\
1 & 0 & 0 & 0 \\
0 & 1 & 0 & 0
\end{array}\right) .
\end{aligned}
$$

These obey the relations

$$
\left[\mathbf{t}_{i}^{ \pm}, \mathbf{t}_{j}^{ \pm}\right]=\mp \epsilon_{i j k} \mathbf{t}_{k}^{ \pm}, \quad \operatorname{tr}\left(\mathbf{t}_{i}^{+} \mathbf{t}_{j}^{-}\right)=0, \quad \operatorname{tr}\left(\mathbf{t}_{i}^{ \pm}, \mathbf{t}_{j}^{ \pm}\right)=-4 \delta_{i j}, \quad(i, j, k=1,2,3)
$$

\section{Finding the 2-DGM Solution}

The string action (8), when restricted to the sector $\mathbb{R} \times S^{5}$, with $Y_{0}=t$, can be rewritten as the action for a sigma model on the coset space $S U(4) / S p(2) \approx S^{5}$. An element of this coset will obey

$$
\begin{gathered}
\mathbf{g}^{\dagger} \mathbf{g}=\mathbf{1}, \\
\mathcal{J} \mathbf{g} \mathcal{J}^{-1}=\mathbf{g}^{T},
\end{gathered}
$$

where

$$
\mathcal{J}=\left(\begin{array}{cc}
0 & \mathbf{1} \\
-\mathbf{1} & 0
\end{array}\right)
$$

A convenient parametrisation that makes contact with $(8,11)$ is

$$
\mathbf{g}=\left(\begin{array}{cccc}
Z_{1} & Z_{2} & 0 & Z_{3} \\
-\bar{Z}_{2} & \bar{Z}_{1} & -Z_{3} & 0 \\
0 & \bar{Z}_{3} & Z_{1} & -\bar{Z}_{2} \\
-\bar{Z}_{3} & 0 & Z_{2} & \bar{Z}_{1}
\end{array}\right)
$$

The first coset condition becomes

$$
\left|Z_{1}\right|^{2}+\left|Z_{2}\right|^{2}+\left|Z_{3}\right|^{2}=1
$$


and the second is trivially verified.

A simple solution for the equations of motion is

$$
\mathbf{g}_{0}=\operatorname{diag}\left(e^{i t}, e^{-i t}, e^{i t}, e^{-i t}\right) .
$$

This corresponds to a point particle moving along the equator of $S^{5}$ : the BMN string (14).

The correspondent wave-function can then be computed,

$$
\Psi_{0}=\operatorname{diag}\left(e^{i Z}, e^{-i Z}, e^{i Z}, e^{-i Z}\right)
$$

with

$$
Z(\lambda)=\frac{x_{+}}{\lambda+1}+\frac{x_{-}}{\lambda-1}, \quad x_{ \pm}=\frac{1}{2}(x \pm t),
$$

$(x, t)$ the worldsheet string coordinates, and $\lambda$ a complex parameter.

In the description of the solitons it is convenient to define the coordinates $(u, v)$ by

$$
u=i[Z(\lambda)-Z(\bar{\lambda})], \quad v=Z(\lambda)+Z(\bar{\lambda})-t
$$

If we write $\lambda=r e^{i p / 2}$, and make $p=\pi-\delta$ these become

$$
u=\frac{2 r\left(1+r^{2}\right) \cos \left(\frac{\delta}{2}\right)}{1+r^{4}+2 r^{2} \cos (\delta)}[x-V t], \quad v=\frac{2 r\left(r^{2}-1\right) \sin \left(\frac{\delta}{2}\right)}{1+r^{4}+2 r^{2} \cos (\delta)}\left[x-\frac{t}{V}\right],
$$

with

$$
V=\frac{2 r}{r^{2}+1} \sin \left(\frac{\delta}{2}\right)
$$

These are the functions (22-23) defined on the main text.

Using the dressing method, the 1-soliton wave function can be computed by dressing the vacuum wave function,

$$
\Psi_{1}=\chi_{1} \Psi_{0}
$$

with the dressing factor

$$
\chi_{1}=\mathbf{1}+\frac{\lambda_{1}-\bar{\lambda}_{1}}{\lambda-\lambda_{1}} \mathcal{P}_{0}+\frac{1 / \bar{\lambda}_{1}-1 / \lambda_{1}}{\lambda-1 / \bar{\lambda}_{1}} \mathcal{Q}_{0}
$$

where $\lambda_{1}=r_{1} e^{i \frac{p_{1}}{2}}$ is the complex parameter that classifies the properties of the soliton solution (such as its momentum and angular momentum). $\mathcal{P}_{0}$ and $\mathcal{Q}_{0}$ are projectors that are determined from the vacuum wave-function:

$$
\begin{gathered}
\mathcal{P}_{1}=\frac{\vec{a}_{\mathcal{P}_{1}} \otimes \vec{b}_{\mathcal{P}_{1}}}{\vec{a}_{\mathcal{P}_{1}} \cdot \vec{b}_{\mathcal{P}_{1}}}, \quad \mathcal{Q}_{1}=\frac{\vec{a}_{\mathcal{Q}_{1}} \otimes \vec{b}_{\mathcal{Q}_{1}}}{\vec{a}_{\mathcal{Q}_{1}} \cdot \vec{b}_{\mathcal{Q}_{1}}} \\
\vec{a}_{\mathcal{P}_{1}}=\Psi_{0}\left(\bar{\lambda}_{1}\right) w_{1}, \quad \vec{b}_{\mathcal{P}_{1}}=w_{1}^{\dagger}\left[\Psi_{0}\left(\bar{\lambda}_{1}\right)\right]^{\dagger}, \\
\vec{a}_{\mathcal{Q}_{1}}=\Psi_{0}\left(\lambda_{1}^{-1}\right) \mathcal{J} \bar{w}_{1}, \quad \vec{b}_{\mathcal{Q}_{1}}=\mathcal{J}^{-1}\left[\Psi\left(\lambda_{1}^{-1}\right)\right]^{\dagger} .
\end{gathered}
$$


$w_{1}$ is a 4 -vector that determines the polarisation of the giant magnon. The soliton solutions (37) with parameters (38) can be generated separately from the polarisations

$$
w_{1}=\left(\begin{array}{llll}
i & 1 & 0 & 0
\end{array}\right)^{T}, \quad w_{2}=\left(\begin{array}{c}
i e^{-D / 2+i\left(\psi_{+}+\psi_{-}-\theta_{0}\right) / 2} \cos \alpha_{-} \\
e^{D / 2+i\left(-\psi_{+}+\psi_{-}+\theta_{0}\right) / 2} \cos \alpha_{+} \\
-i e^{-D / 2+i\left(-\psi_{+}-\psi_{-}-\theta_{0}\right) / 2} \sin \alpha_{-} \\
e^{D / 2+i\left(\psi_{+}-\psi_{-}+\theta_{0}\right) / 2} \sin \alpha_{+}
\end{array}\right)
$$

by taking $\mathbf{g}_{1}=\Psi_{1}[\lambda=0]$.

We wish to identify a 2-DGM solution that at $t=0$ resembles, near the vicinity of each soliton, the direct sum of the solutions (37). If we naively pick the solution

$$
\mathbf{g}_{2}=\chi_{2}\left[\lambda=0 ; w_{2}\right] \chi_{1}\left[\lambda=0 ; w_{1}\right] \mathbf{g}_{1}
$$

we find out that separation between the solitons is no longer $d$ but that it receives a leading order correction due to a time delay in the scattering. In such solution, the solitons are not positioned at $t=0$ as those in (37-38). Further more, the solution $\mathbf{g}_{2}$ appears to be rotated, the first soliton not being fixed at $Z_{3}=0$. By changing the polarisations and inducing a global rotation in $\mathbf{g}_{2}$, we can generate a two soliton solution $\tilde{\mathbf{g}}_{2}$ that resembles the direct sum of the solitons (37-38) at $t=0$, in the neighbourhood of the centre of each DGM. This solution can be formally constructed from the polarizations:

$$
\tilde{w}_{1}=\left(\begin{array}{llll}
i e^{-\frac{\Delta_{-}}{2}} & e^{\frac{\Delta_{-}}{2}} & 0 & 0
\end{array}\right)^{T}, \quad \tilde{w}_{2}=\left(\begin{array}{c}
i e^{-\left(D-\Delta_{+}\right) / 2+i\left(\psi_{+}+\psi_{-}-\theta_{0}\right) / 2} \cos \alpha_{-} \\
e^{\left(D-\Delta_{+}\right) / 2+i\left(-\psi_{+}+\psi_{-}+\theta_{0}\right) / 2} \cos \alpha_{+} \\
-i e^{-\left(D-\Delta_{+}\right) / 2+i\left(-\psi_{+}-\psi_{-}-\theta_{0}\right) / 2} \sin \alpha_{-} \\
e^{\left(D-\Delta_{+}\right) / 2+i\left(\psi_{+}-\psi_{-}+\theta_{0}\right) / 2} \sin \alpha_{+}
\end{array}\right),
$$

with $\Delta_{ \pm}$given by

$$
\Delta_{ \pm}= \pm \frac{1}{2} \log \left(\frac{A}{B_{ \pm}}\right)
$$

and

$$
\begin{gathered}
A=\left(r_{1}+r_{2}\right)^{2}\left(1+r_{1} r_{2}\right)^{2} \\
B_{ \pm}=r_{2}^{2}+r_{1}^{4} r_{2}^{2}+r_{1}\left(r_{2}+r_{2}^{3}\right)+r_{1}^{3}\left(r_{2}+r_{2}^{3}\right)+r_{1}^{2}\left(1+r_{2}^{4}\right) \\
-r_{1} r_{2}\left[2\left(-1+r_{1}^{2}\right)\left(-1+r_{2}^{2}\right) \cos \left(2 \alpha_{ \pm}\right) \cos ^{2}\left(\frac{\delta}{2}\right)+\left(1+4 r_{1} r_{2}+r_{2}^{2}+r_{1}^{2}\left(1+r_{2}^{2}\right)\right) \cos \delta\right] .
\end{gathered}
$$

These expressions simplify to (74) in the non-relativistic regime. The solution will then be given, up to a global rotation, by

$$
\tilde{\mathbf{g}}_{2}=\chi_{\tilde{w}_{2}}(0) \chi_{\tilde{w}_{1}}(0) \mathbf{g}_{0}
$$


We don't need to determine the explicit form of $\tilde{\mathbf{g}}_{2}$, only its NLO corrections (the LO correction is compensated already by our particular choice of the polarizations).

Let us turn back to $\mathbf{g}_{2}$ again. For well separated solitons, $D>>1$, we can expand the dressing factor in powers of $e^{-D}$

$$
\chi_{2}=\chi_{2}^{(0)}+\chi_{2}^{(1)} e^{-D}+\mathcal{O}\left[e^{-2 D}\right] .
$$

We note that the vectors $\vec{a}_{2}, \vec{b}_{2}$ exhibit the following dependence on $D$,

$$
\vec{a}_{2}=\vec{a}_{2}^{+} e^{\frac{D}{2}}+\vec{a}_{2}^{-} e^{-\frac{D}{2}}, \quad \vec{b}_{2}=\vec{b}_{2}^{+} e^{\frac{D}{2}}+\vec{b}_{2}^{-} e^{-\frac{D}{2}}
$$

By setting

$$
\mathcal{P}_{2}=\frac{\mathcal{N}_{\mathcal{P} 2}}{\mathcal{D}_{\mathcal{P} 2}}, \quad \mathcal{Q}_{2}=\frac{\mathcal{N}_{\mathcal{Q} 2}}{\mathcal{D}_{\mathcal{Q} 2}},
$$

this translates into

$$
\begin{gathered}
\mathcal{N}_{2} \equiv \vec{a}_{2} \otimes \vec{b}_{2}=\mathcal{N}_{2}^{+} e^{D}+\mathcal{N}_{2}^{0}+\mathcal{N}_{2}^{-} e^{-D} \\
\mathcal{D}_{2} \equiv \vec{a}_{2} \cdot \vec{b}_{2}=\mathcal{D}_{2}^{+} e^{D}+\mathcal{D}_{2}^{0}+\mathcal{D}_{2}^{-} e^{-D}
\end{gathered}
$$

where

$$
\begin{gathered}
\mathcal{N}_{2}^{ \pm} \equiv \vec{a}_{2}^{ \pm} \otimes \vec{b}_{2}^{ \pm}, \quad \mathcal{N}_{2}^{0}=\vec{a}_{2}^{+} \otimes \vec{b}_{2}^{-}+\vec{a}_{2}^{-} \otimes \vec{b}_{2}^{+}, \\
\mathcal{D}_{2}^{ \pm} \equiv \vec{a}_{2}^{ \pm} \cdot \vec{b}_{2}^{ \pm}, \quad \mathcal{N}_{2}^{0}=\vec{a}_{2}^{+} \cdot \vec{b}_{2}^{-}+\vec{a}_{2}^{-} \cdot \vec{b}_{2}^{+} .
\end{gathered}
$$

By expanding in $e^{-D}$ the projectors, we have that

$$
\begin{gathered}
\mathcal{P}_{2}^{(0)}=\frac{\mathcal{N}_{\mathcal{P} 2}^{+}}{\mathcal{D}_{\mathcal{P} 2}^{+}}, \quad \mathcal{Q}_{2}^{(0)}=\frac{\mathcal{N}_{\mathcal{Q} 2}^{+}}{\mathcal{D}_{\mathcal{Q} 2}^{+}}, \quad \text { at LO } \\
\mathcal{P}_{2}^{(1)}=\frac{1}{\mathcal{D}_{\mathcal{P} 2}^{+}}\left(\mathcal{N}_{\mathcal{P} 2}^{0}-\frac{\mathcal{D}_{\mathcal{P} 2}^{0}}{\mathcal{D}_{\mathcal{P} 2}^{+}} \mathcal{N}_{\mathcal{P} 2}^{+}\right), \quad \mathcal{Q}_{2}^{(1)}=\frac{1}{\mathcal{D}_{\mathcal{Q} 2}^{+}}\left(\mathcal{N}_{\mathcal{Q} 2}^{0}-\frac{\mathcal{D}_{\mathcal{Q} 2}^{0}}{\mathcal{D}_{\mathcal{Q} 2}^{+}} \mathcal{N}_{\mathcal{Q} 2}^{+}\right), \quad \text { at NLO }
\end{gathered}
$$

From where the terms in (72) can be computed. The LO correction will be responsible for a shift in the positions of the solitons at time $t=0$, in relation to the direct sum. The shifts will be given by

$$
\Delta X_{1}=\tan \left(\frac{\delta}{2}\right) \frac{\Delta_{-}}{\gamma_{1}^{2} V_{1}} \Delta X_{2}=-\tan \left(\frac{\delta}{2}\right) \frac{\Delta_{+}}{\gamma_{2}^{2} V_{2}}
$$

where $\gamma_{j}=\left(1-V_{j}^{2}\right)^{\frac{1}{2}}$, and $\Delta_{ \pm}$can be approximated, in the non-relativist regime $\delta<<1$, by

$$
\exp \left( \pm 2 \Delta_{ \pm}\right) \approx \frac{\left(m_{1}+m_{2}\right)^{2}}{\left(\vec{J}_{1}^{ \pm}-\vec{J}_{2}^{ \pm}\right)^{2}}
$$

$\vec{J}_{j}^{ \pm}$being the angular momenta of the free solutions (37). The time delay is proportional to $\Delta_{+}-\Delta_{-}$. As previously mentioned, the polarisations (66) should be modified to accommodate these changes. 
This is easily done by the change $D \rightarrow D-\Delta_{+}$on $w_{2}$ and by taking $w_{1}=\left(i e^{-\frac{\Delta_{-}}{2}}, e^{\frac{\Delta_{-}}{2}}, 0,0\right)$. This justifies the selection of the polarizations $\tilde{w}_{1}$ and $\tilde{w}_{2}$ in the construction of $\tilde{\mathbf{g}}_{2}$.

Not only the positions of the solitons have been changed due to LO corrections, but the solution appears rotated as well. The relative orientation of the two solitons is kept, but a global rotation was induced by the application of the dressing method. To undo this, we need to choose the rotation that fixes $Z_{3}=0$ at LO in the neighbourhood of the origin. The rotation is easily found to be

$$
\begin{aligned}
& \tilde{Z}_{2}^{(0)}=\bar{C}_{2} Z_{2}^{(0)}+\bar{C}_{3} Z_{3}^{(0)}, \\
& \tilde{Z}_{3}^{(0)}=C_{3} Z_{2}^{(0)}-C_{2} Z_{3}^{(0)},
\end{aligned}
$$

with

$$
\begin{aligned}
C_{2} & =e^{-\Delta_{-}} \frac{\left[r_{2}-r_{1}^{2} r_{2}+r_{1}\left(-1+r_{2}^{2}\right) \cos \left(2 \alpha_{+}\right)\right]\left(1+\cos \delta_{1}\right)+i\left(r_{1}+r_{2}\right)\left(1+r_{1} r_{2}\right) \sin \delta_{1}}{\left(1+e^{i \delta_{1}}\right)\left(r_{1}+r_{2}\right)\left(1+r_{1} r_{2}\right)}, \\
C_{3} & =e^{-\Delta_{-}} \frac{e^{-i\left(\delta_{1}+\psi_{+}\right)}\left(1+e^{i \delta_{1}}\right) r_{1}\left(-1+r_{2}^{2}\right) \cos \alpha_{+} \sin \alpha_{+}}{\left(r_{1}+r_{2}\right)\left(1+r_{1} r_{2}\right)},
\end{aligned}
$$

with $\left|C_{2}\right|^{2}+\left|C_{3}\right|^{2}=1 . Z_{1}$ remains unmodified. With this rotation in place (applied to the NLO as well), and with the polarisation shifts, the 2-DGM solution thus obtained can be approximated at LO, in the neighbourhood of each soliton, by the sum of the two free solutions (37). In particular, for $\delta Z_{2}^{(1)}$ we find ${ }^{3}$,

$$
\begin{gathered}
\delta Z_{2}^{(1)}=-\frac{i e^{-u_{2}-i\left(v_{2}+\alpha_{-}+\alpha_{+}+\delta+i \psi_{+}\right)}\left(1+e^{i \delta}\right)}{4\left(1+e^{2 u_{1}}\right)^{2}\left(r_{1}+r_{2}\right)^{2}\left(1+r_{1} r_{2}\right)^{2}} \\
\quad \times\left[-e^{2 i \psi_{+}}\left(r_{1}+r_{2}\right)^{2}+e^{2 i\left(\alpha_{-}+\psi_{+}\right)}\left(r_{1}+r_{2}\right)^{2}+e^{2 i\left(\alpha_{+}+\psi_{+}\right)}\left(r_{1}+r_{2}\right)^{2}\right. \\
\quad-e^{2 i\left(\alpha_{-}+\alpha_{+}+\psi_{+}\right)}\left(r_{1}+r_{2}\right)^{2}-e^{2\left(u_{1}+i\left(v_{1}+v_{2}\right)\right)} r_{1} r_{2}\left(r_{1}+r_{2}\right)^{2} \\
+e^{2\left(u_{1}+i\left(v_{1}+v_{2}+\alpha_{-}\right)\right)} r_{1} r_{2}\left(r_{1}+r_{2}\right)^{2}+e^{2\left(u_{1}+i\left(v_{1}+v_{2}+\alpha_{+}\right)\right)} r_{1} r_{2}\left(r_{1}+r_{2}\right)^{2} \\
-e^{2\left(u_{1}+i\left(v_{1}+v_{2}+\alpha_{-}+\alpha_{+}\right)\right)} r_{1} r_{2}\left(r_{1}+r_{2}\right)^{2}-e^{2\left(u_{1}+i\left(v_{1}+v_{2}+\delta\right)\right)} r_{1} r_{2}\left(r_{1}+r_{2}\right)^{2} \\
-2 e^{2 u_{1}+i\left(2 v_{1}+2 v_{2}+\delta\right)} r_{1} r_{2}\left(r_{1}+r_{2}\right)^{2}+e^{2\left(u_{1}+i\left(v_{1}+v_{2}+\alpha_{-}+\delta\right)\right)} r_{1} r_{2}\left(r_{1}+r_{2}\right)^{2} \\
+2 e^{2 u_{1}+i\left(2 v_{1}+2 v_{2}+2 \alpha_{-}+\delta\right)} r_{1} r_{2}\left(r_{1}+r_{2}\right)^{2}+e^{2\left(u_{1}+i\left(v_{1}+v_{2}+\alpha_{+}+\delta\right)\right)} r_{1} r_{2}\left(r_{1}+r_{2}\right)^{2} \\
-e^{2\left(u_{1}+i\left(v_{1}+v_{2}+\alpha_{-}+\alpha_{+}+\delta\right)\right)} r_{1} r_{2}\left(r_{1}+r_{2}\right)^{2}+2 e^{2 u_{1}+i\left(2 v_{1}+2 v_{2}+2 \alpha_{+}+\delta\right)} r_{1} r_{2}\left(r_{1}+r_{2}\right)^{2} \\
-2 e^{2 u_{1}+i\left(2 v_{1}+2 v_{2}+2 \alpha_{-}+2 \alpha_{+}+\delta\right)} r_{1} r_{2}\left(r_{1}+r_{2}\right)^{2}+2 e^{i\left(\delta+2 \psi_{+}\right)} r_{1} r_{2}\left(r_{1}+r_{2}\right)^{2} \\
-2 e^{i\left(2 \alpha_{-}+\delta+2 \psi_{+}\right)} r_{1} r_{2}\left(r_{1}+r_{2}\right)^{2}-2 e^{i\left(2 \alpha_{+}+\delta+2 \psi_{+}\right)} r_{1} r_{2}\left(r_{1}+r_{2}\right)^{2}+2 e^{i\left(2 \alpha_{-}+2 \alpha_{+}+\delta+2 \psi_{+}\right)} r_{1} r_{2}\left(r_{1}+r_{2}\right)^{2} \\
-e^{2 i\left(\delta+\psi_{+}\right)} r_{1}^{2} r_{2}^{2}\left(r_{1}+r_{2}\right)^{2}+e^{2 i\left(\alpha_{-}+\delta+\psi_{+}\right)} r_{1}^{2} r_{2}^{2}\left(r_{1}+r_{2}\right)^{2}+\cdots
\end{gathered}
$$

\footnotetext{
${ }^{3}$ we have absorbed $\theta_{0}$ in $v_{2}$ in these expressions.
} 


$$
\begin{aligned}
& \cdots-e^{2 u_{1}+2 i \psi_{+}}\left(r_{1}+r_{2}\right)^{2}\left(1+r_{1} r_{2}\right)+e^{2\left(u_{1}+i\left(\alpha_{-}+\psi_{+}\right)\right)}\left(r_{1}+r_{2}\right)^{2}\left(1+r_{1} r_{2}\right)+e^{2\left(u_{1}+i\left(\alpha_{+}+\psi_{+}\right)\right)}\left(r_{1}+r_{2}\right)^{2}\left(1+r_{1} r_{2}\right) \\
& -e^{2\left(u_{1}+i\left(\alpha_{-}+\alpha_{+}+\psi_{+}\right)\right)}\left(r_{1}+r_{2}\right)^{2}\left(1+r_{1} r_{2}\right) \\
& -e^{2\left(u_{1}+i\left(\delta+\psi_{+}\right)\right)} r_{1} r_{2}\left(r_{1}+r_{2}\right)^{2}\left(1+r_{1} r_{2}\right)+e^{2\left(u_{1}+i\left(\alpha_{-}+\delta+\psi_{+}\right)\right)} r_{1} r_{2}\left(r_{1}+r_{2}\right)^{2}\left(1+r_{1} r_{2}\right) \\
& +e^{2\left(u_{1}+i\left(\alpha_{+}+\delta+\psi_{+}\right)\right)} r_{1} r_{2}\left(r_{1}+r_{2}\right)^{2}\left(1+r_{1} r_{2}\right)-e^{2\left(u_{1}+i\left(\alpha_{-}+\alpha_{+}+\delta+\psi_{+}\right)\right)} r_{1} r_{2}\left(r_{1}+r_{2}\right)^{2}\left(1+r_{1} r_{2}\right) \\
& +e^{2 i\left(v_{2}+\delta+\psi_{+}\right)} r_{1}^{2}\left(1+r_{1} r_{2}\right)^{2}+e^{2 i\left(v_{2}+\alpha_{-}+\delta+\psi_{+}\right)} r_{1}^{2}\left(1+r_{1} r_{2}\right)^{2} \\
& +e^{2 i\left(v_{2}+\alpha_{+}+\delta+\psi_{+}\right)} r_{1}^{2}\left(1+r_{1} r_{2}\right)^{2}+e^{2 i\left(v_{2}+\alpha_{-}+\alpha_{+}+\delta+\psi_{+}\right)} r_{1}^{2}\left(1+r_{1} r_{2}\right)^{2} \\
& +e^{2 u_{1}+2 i v_{1}} r_{1} r_{2}\left(1+r_{1} r_{2}\right)^{2}+e^{2\left(u_{1}+i\left(v_{1}+\alpha_{-}\right)\right)} r_{1} r_{2}\left(1+r_{1} r_{2}\right)^{2} \\
& +e^{2\left(u_{1}+i\left(v_{1}+\alpha_{+}\right)\right)} r_{1} r_{2}\left(1+r_{1} r_{2}\right)^{2}+e^{2\left(u_{1}+i\left(v_{1}+\alpha_{-}+\alpha_{+}\right)\right)} r_{1} r_{2}\left(1+r_{1} r_{2}\right)^{2} \\
& +e^{2\left(u_{1}+i\left(v_{1}+\delta\right)\right)} r_{1} r_{2}\left(1+r_{1} r_{2}\right)^{2}+2 e^{2 u_{1}+i\left(2 v_{1}+\delta\right)} r_{1} r_{2}\left(1+r_{1} r_{2}\right)^{2}+e^{2\left(u_{1}+i\left(v_{1}+\alpha-+\delta\right)\right)} r_{1} r_{2}\left(1+r_{1} r_{2}\right)^{2} \\
& +2 e^{2 u_{1}+i\left(2 v_{1}+2 \alpha_{-}+\delta\right)} r_{1} r_{2}\left(1+r_{1} r_{2}\right)^{2}+e^{2\left(u_{1}+i\left(v_{1}+\alpha_{+}+\delta\right)\right)} r_{1} r_{2}\left(1+r_{1} r_{2}\right)^{2} \\
& +e^{2\left(u_{1}+i\left(v_{1}+\alpha_{-}+\alpha_{+}+\delta\right)\right)} r_{1} r_{2}\left(1+r_{1} r_{2}\right)^{2}+2 e^{2 u_{1}+i\left(2 v_{1}+2 \alpha_{+}+\delta\right)} r_{1} r_{2}\left(1+r_{1} r_{2}\right)^{2} \\
& +2 e^{2 u_{1}+i\left(2 v_{1}+2 \alpha_{-}+2 \alpha_{+}+\delta\right)} r_{1} r_{2}\left(1+r_{1} r_{2}\right)^{2}- \\
& -2 e^{i\left(2 v_{2}+\delta+2 \psi_{+}\right)} r_{1} r_{2}\left(1+r_{1} r_{2}\right)^{2}-2 e^{i\left(2 v_{2}+2 \alpha_{-}+\delta+2 \psi_{+}\right)} r_{1} r_{2}\left(1+r_{1} r_{2}\right)^{2} \\
& -2 e^{i\left(2 v_{2}+2 \alpha_{+}+\delta+2 \psi_{+}\right)} r_{1} r_{2}\left(1+r_{1} r_{2}\right)^{2}-2 e^{i\left(2 v_{2}+2 \alpha_{-}+2 \alpha_{+}+\delta+2 \psi_{+}\right)} r_{1} r_{2}\left(1+r_{1} r_{2}\right)^{2} \\
& +e^{2 i\left(v_{2}+\psi_{+}\right)} r_{2}^{2}\left(1+r_{1} r_{2}\right)^{2}+e^{2 i\left(v_{2}+\alpha_{-}+\psi_{+}\right)} r_{2}^{2}\left(1+r_{1} r_{2}\right)^{2}+e^{2 i\left(v_{2}+\alpha_{+}+\psi_{+}\right)} r_{2}^{2}\left(1+r_{1} r_{2}\right)^{2} \\
& +e^{2 i\left(v_{2}+\alpha_{-}+\alpha_{+}+\psi_{+}\right)} r_{2}^{2}\left(1+r_{1} r_{2}\right)^{2}+e^{2\left(u_{1}+i\left(v_{2}+\delta+\psi_{+}\right)\right)} r_{1}\left(r_{1}+r_{2}\right)\left(1+r_{1} r_{2}\right)^{2} \\
& +e^{2\left(u_{1}+i\left(v_{2}+\alpha_{-}+\delta+\psi_{+}\right)\right)} r_{1}\left(r_{1}+r_{2}\right)\left(1+r_{1} r_{2}\right)^{2}+e^{2\left(u_{1}+i\left(v_{2}+\alpha_{+}+\delta+\psi_{+}\right)\right)} r_{1}\left(r_{1}+r_{2}\right)\left(1+r_{1} r_{2}\right)^{2} \\
& +e^{2\left(u_{1}+i\left(v_{2}+\alpha_{-}+\alpha_{+}+\delta+\psi_{+}\right)\right)} r_{1}\left(r_{1}+r_{2}\right)\left(1+r_{1} r_{2}\right)^{2}+e^{2\left(u_{1}+i\left(v_{2}+\psi_{+}\right)\right)} r_{2}\left(r_{1}+r_{2}\right)\left(1+r_{1} r_{2}\right)^{2} \\
& +e^{2\left(u_{1}+i\left(v_{2}+\alpha_{-}+\psi_{+}\right)\right)} r_{2}\left(r_{1}+r_{2}\right)\left(1+r_{1} r_{2}\right)^{2}+e^{2\left(u_{1}+i\left(v_{2}+\alpha_{+}+\psi_{+}\right)\right)} r_{2}\left(r_{1}+r_{2}\right)\left(1+r_{1} r_{2}\right)^{2} \\
& +e^{2\left(u_{1}+i\left(v_{2}+\alpha_{-}+\alpha_{+}+\psi_{+}\right)\right)} r_{2}\left(r_{1}+r_{2}\right)\left(1+r_{1} r_{2}\right)^{2}+e^{2 u_{1}+i\left(\delta+2 \psi_{+}\right)}\left(r_{1}+r_{2}\right)^{2}\left(1+r_{1} r_{2}\right)^{2} \\
& +e^{4 u_{1}+i\left(\delta+2 \psi_{+}\right)}\left(r_{1}+r_{2}\right)^{2}\left(1+r_{1} r_{2}\right)^{2}-e^{2 u_{1}+i\left(2 v_{2}+\delta+2 \psi_{+}\right)}\left(r_{1}+r_{2}\right)^{2}\left(1+r_{1} r_{2}\right)^{2} \\
& -e^{4 u_{1}+i\left(2 v_{2}+\delta+2 \psi_{+}\right)}\left(r_{1}+r_{2}\right)^{2}\left(1+r_{1} r_{2}\right)^{2} \\
& -e^{2 u_{1}+i\left(2 \alpha_{-}+\delta+2 \psi_{+}\right)}\left(r_{1}+r_{2}\right)^{2}\left(1+r_{1} r_{2}\right)^{2}-e^{4 u_{1}+i\left(2 \alpha_{-}+\delta+2 \psi_{+}\right)}\left(r_{1}+r_{2}+r_{1}^{2} r_{2}+r_{1} r_{2}^{2}\right)^{2} \\
& -e^{2 u_{1}+i\left(2 v_{2}+2 \alpha_{-}+\delta+2 \psi_{+}\right)}\left(r_{1}+r_{2}\right)^{2}\left(1+r_{1} r_{2}\right)^{2}-e^{4 u_{1}+i\left(2 v_{2}+2 \alpha_{-}+\delta+2 \psi_{+}\right)}\left(r_{1}+r_{2}\right)^{2}\left(1+r_{1} r_{2}\right)^{2} \\
& -e^{2 u_{1}+i\left(2 \alpha_{+}+\delta+2 \psi_{+}\right)}\left(r_{1}+r_{2}+r_{1}^{2} r_{2}+r_{1} r_{2}^{2}\right)^{2} \\
& -e^{4 u_{1}+i\left(2 \alpha_{+}+\delta+2 \psi_{+}\right)}\left(r_{1}+r_{2}\right)^{2}\left(1+r_{1} r_{2}\right)^{2}-e^{2 u_{1}+i\left(2 v_{2}+2 \alpha_{+}+\delta+2 \psi_{+}\right)}\left(r_{1}+r_{2}\right)^{2}\left(1+r_{1} r_{2}\right)^{2} \\
& -e^{4 u_{1}+i\left(2 v_{2}+2 \alpha_{+}+\delta+2 \psi_{+}\right)}\left(r_{1}+r_{2}\right)^{2}\left(1+r_{1} r_{2}\right)^{2} \\
& +e^{2 u_{1}+i\left(2 \alpha_{-}+2 \alpha_{+}+\delta+2 \psi_{+}\right)}\left(r_{1}+r_{2}\right)^{2}\left(1+r_{1} r_{2}\right)^{2}+e^{4 u_{1}+i\left(2 \alpha_{-}+2 \alpha_{+}+\delta+2 \psi_{+}\right)}\left(r_{1}+r_{2}\right)^{2}\left(1+r_{1} r_{2}\right)^{2} \\
& -e^{2 u_{1}+i\left(2 v_{2}+2 \alpha_{-}+2 \alpha_{+}+\delta+2 \psi_{+}\right)}\left(r_{1}+r_{2}\right)^{2}\left(1+r_{1} r_{2}\right)^{2} \\
& \left.-e^{4 u_{1}+i\left(2 v_{2}+2 \alpha_{-}+2 \alpha_{+}+\delta+2 \psi_{+}\right)}\left(r_{1}+r_{2}\right)^{2}\left(1+r_{1} r_{2}\right)^{2}\right] \text {. }
\end{aligned}
$$


It is straightforward to check that, for $\delta<<1$ and $u_{1} \approx 0$,

$$
\begin{aligned}
\left(\frac{\delta Z_{2}^{(1)}}{Z_{2}^{(1)}}\right)_{x=\omega^{(1)} t}=4 i r_{1} r_{2}\left\{\frac{1}{\left(r_{1}+r_{2}\right)^{2}} \cos \alpha_{-}\right. & \cos \alpha_{+} \sin \left(v_{1}-v_{2}-\psi_{+}\right) \\
& \left.+\frac{1}{\left(1+r_{1} r_{2}\right)^{2}} \sin \alpha_{-} \sin \alpha_{+} \sin \left(v_{1}+v_{2}-\psi_{+}\right)\right\}
\end{aligned}
$$

\section{References}

[1] J. Goldstone and R. Jackiw, Phys. Rev. D 11 (1975) 1486.

[2] R. F. Dashen, B. Hasslacher and A. Neveu, Phys. Rev. D 10 (1974) 4114.

R. F. Dashen, B. Hasslacher and A. Neveu, Phys. Rev. D 10 (1974) 4130.

R. F. Dashen, B. Hasslacher and A. Neveu, Phys. Rev. D 10 (1974) 4138.

[3] L. D. Faddeev and V. E. Korepin, Phys. Rept. 42 (1978) 1.

[4] S. R. Coleman, Phys. Rev. D 11 (1975) 2088

[5] C. Montonen and D. I. Olive, Phys. Lett. B 72, 117 (1977).

[6] G. S. Adkins, C. R. Nappi and E. Witten, Nucl. Phys. B 228, 552 (1983).

[7] D. M. Hofman and J. M. Maldacena, J. Phys. A 39 (2006) 13095 [arXiv:hep-th/0604135].

[8] I. Aniceto and A. Jevicki, arXiv:0810.4548 [hep-th].

[9] N. S. Manton, Nucl. Phys. B 150 (1979) 397.

[10] G. Bowtell and A. Stuart, Phys. Rev. D 15 (1977) 3581

[11] G. Bowtell and A. Stuart, J. Math. Phys. 24 (1983) 969

[12] J. Perring and T. Skyrme Nucl. Phys. 31 (1962) 550

[13] R. Rajaraman, Phys. Rev. D 15 (1977) 2866

[14] S. N. M. Ruijsenaars and H. Schneider, Annals Phys. 170, 370 (1986).

[15] O. Babelon and D. Bernard Phys. Lett. B 317 (1993) 363

[16] N. Dorey, D. M. Hofman and J. M. Maldacena, Phys. Rev. D 76 (2007) 025011 [arXiv:hepth/0703104]. 
[17] K. Pohlmeyer, Commun. Math. Phys. 46 (1976) 207.

K. Pohlmeyer and K. H. Rehren, J. Math. Phys. 20 (1979) 2628.

[18] D. E. Berenstein, J. M. Maldacena and H. S. Nastase, JHEP 0204 (2002) 013 [arXiv:hepth/0202021].

[19] H. Y. Chen, N. Dorey and K. Okamura, JHEP 0609 (2006) 024 [arXiv:hep-th/0605155].

[20] M. Spradlin and A. Volovich, JHEP 0610 (2006) 012 [arXiv:hep-th/0607009].

[21] C. Kalousios, M. Spradlin and A. Volovich, JHEP 0703 (2007) 020 [arXiv:hep-th/0611033]. 